\title{
SOLIDIFICATION FRONT TILT ANGLE EFFECT ON POTENTIAL NUCLEATION SITES FOR FRECKLING IN THE REMELT OF NI-BASE SUPERALLOYS
}

\author{
Jairo Valdes ${ }^{1,2}$, Xingbo Liu ${ }^{1}$, Paul King ${ }^{3}$, Christopher Cowen ${ }^{3}$, Paul Jablonski ${ }^{3}$ \\ ${ }^{1}$ Mechanical \& Aerospace Engineering Department, West Virginia University, \\ Morgantown, WV 26506-6106, USA \\ ${ }^{2}$ Escuela de Ingeniería Mecánica, Universidad del Valle, Cali, COLOMBIA \\ ${ }^{3}$ National Energy Technology Laboratory, Albany, OR 97321, USA
}

Keywords: Tilt angle, Freckle, Ra Number, Permeability

\begin{abstract}
By considering the mushy layer as a porous media with variable permeability, a Rayleigh number based freckling criterion was developed from the Flemings ratio between the magnitude of the interdendritic flow velocity and the solidification rate. The proposed form includes the effect of the tilt angle by preserving the anisotropic nature of the permeability tensor throughout the derivation and uses Poirier's experimentally determined functional forms for the parallel and perpendicular components. The proposed form of Rayleigh number criterion was found to provide better resolution when evaluated against available experimental data in the literature. Especially, it showed that the nucleation of channels in the mushy layer leading to freckles is equally probable in the proximity of the tips of the dendrites or deeper in the mushy layer, for example at approximately 0.7 liquid fraction and 0.4 liquid fraction respectively, depending on the angle of tilt of the solidification front.
\end{abstract}

\section{Introduction}

Large Ni-base superalloy ingots required for increased efficiency gas turbines in power applications have a marked tendency to form macrosegregation defects as freckles. These defects are discontinuities in the material that act as stress concentration points making it highly sensitive to fatigue crack growth under thermal and mechanical cyclic loads. They cannot be removed by thermo-mechanical treatment making the ingot unsuitable and should be scrapped. Advances in quality assurance of electrodes and improved control of the VAR process have contributed to reduce the incidence of freckles in large ingots ${ }^{1}$ but freckles are still found when the VAR stability is perturbed by transients in the arc melting conditions that affect the macroscopic transfer of mass heat and momentum. ${ }^{2}$ It is necessary to continue efforts for better 
understanding of the mechanisms of nucleation and growth of freckles during VAR process in order to expand the processing window and further increase the diameter of ingots to meet the demands of larger rotor discs for the new generation of gas turbines.

\section{$\underline{\text { Nucleation sites and freckle formation mechanism }}$}

Since near four decades ago the freckle formation problem has been studied by researchers in different areas of science and engineering, and it has been understood as a case of thermo-solutal convection in a reactive porous media. ${ }^{3}$ Therefore, there is vast collection of publications on the subject and it is not intended on this work to include a comprehensive literature review, but to include the most relevant antecedents. Consider the case of an ingot cooled from the bottom with heat flow assumed unidirectional, and solidification process occurring with constant upward rate. Once the positive thermal gradient $(\partial \mathrm{T} / \partial \mathrm{z})$ is established and solidification advances, composition and density gradients develop along the mush layer due to partitioning of solute elements. According to the set of solute elements and their partition behavior, the resulting density gradient $(\partial \rho / \partial z)$ can be positive or negative and this defines the conditions by which interactions may occur between the interdendritic liquid and the solid dendrites. If the density gradient is positive, a density inversion condition exists and the buoyancy forces could become strong enough to overcome the impedance to flow offered by the dendritic array. Upward transport of interdendritic liquid will occur with a corresponding concomitant flow of heavier reposition liquid from higher locations. The flow of segregated liquid will remelt the already formed solid while it attains thermodynamical equilibrium with the new surroundings; and the columnar regions with decreased solid fraction, and hence decreased resistance to the flow, eventually turn into chimneys through which plumes of interdendritic liquid are projected into the bulk liquid. Lastly, the remaining chimneys solidify as the defects known as freckles containing low melting point-close to eutectic composition material and porosity. This general mechanism is widely accepted and has been supported by detailed experimental observations and numerical simulations using both analogous transparent systems and metallic alloys. ${ }^{4,5}$ Single crystal alloys with high content of refractory elements are typically susceptible to freckles

formed by this mechanism, as well as some wrought superalloys. ${ }^{6}$ Generalized agreement exists about the convective origin of upward interdendritic liquid flow and its role on formation of vertical freckles, but there is no complete consonance about the specific nucleation sites and 
evolution sequence of the channels leading to their formation. Sample and Hellawell ${ }^{7}$ concluded that channels originate at the growth front and propagate back into the mush. For the nucleation to occur, it is deemed necessary the evolution of double diffusive finger convection cells in a thin liquid layer with inverted density built in top of the mush. Their proposed sequence of nucleation at the mush low solid fraction region and downward growth of the channels has been later supported by numerical simulations and experiments. ${ }^{7,8,9,10,11}$ A major supporting evidence lies on the fact that when externally induced shear movement between the bulk liquid and the top mush layer disrupts the convection cells evolution, freckles are not formed or if they do appear, their distribution is not random, but are confined to the walls of the ingot, where the relative movement is weaker. Differently, linear stability analysis of thermosolutal convection performed by Worster ${ }^{12}$ on 'ideal' mush layers lead to the conclusion that there are two possible modes of convection which can occur independently. The first mode called boundary layer mode corresponds to the double diffusive fingers on the interface mush-bulk liquid, which is understood to leave the fluid in the mush stagnant, while the second convection mode denominated mush layer mode happens within the mush and causes perturbations in the solid fraction leading to formation of chimneys. ${ }^{12,13}$ This hypothesis of nucleation and growth of channels inside the mush layer has also been supported by theoretical and experimental results. ${ }^{14}$, ${ }^{15,16}$ When segregation of the interdendritic liquid builds a negative density gradient in the mush layer, it will be gravitationally stabilizing and the interdendritic liquid will be stratified with no density inversion observed. Whenever the heat flux is not aligned with gravity, the solidification front is not perpendicular to the gravity vector and it has been proposed that the heavier liquid will tend to seep between the dendrites, following a path almost parallel to the liquidus isotherm melting-eroding the dendrites on its path and give origin to the freckles seen on VAR ingots. ${ }^{17,18}$ This proposed mechanism for VAR freckles has been devised mostly from the observed morphology and characterization of freckles in solidified ingots. Regarding the nucleation sites for the formation of channels leading to freckles, Auburtin et al. ${ }^{19}$ used SEM/EDAX to determine freckles average composition and compared it with the segregation profiles to conclude that they formed from a mush region with solid fraction of 0.4 to 0.6 . When compared with the density inversion case, appreciably less detailed information is available regarding the sequence of events leading to the nucleation and growth of freckles in VAR due to heavier interdendritic liquid transport. Usually freckles are found in the mid radius and center of VAR 
ingots, and are more frequently found when the molten metal pool has a steeper 'bowl shape' like the one when the melting rate is high or in ESR ingots. Numerical simulations have corroborated the foundry practice knowledge that abrupt variations on the controlling parameters of VAR induce perturbations in the temperature and composition of the interdendritic liquid that have a strong influence on freckle nucleation and growth. ${ }^{20,21,22}$ Nevertheless, no industrial application of direct simulation of freckles in VAR has been reached. It has been proposed that horizontal directional solidification experiments should better resemble the freckling conditions for VAR ingots. ${ }^{23,}{ }^{24}$ Results showed that freckles can propagate without the influence of the strong electromagnetic Lorentz forces present on the VAR process, indicating that it is feasible that freckles have a nucleation mechanism that not necessarily depends on the bulk liquid convection. A complete understanding of the nucleation and growth of freckles in VAR ingots is required in order to construct accurate predictions and more efficient prevention strategies.

\section{Prediction of freckles formation}

Two major methodology tendencies could be recognized on the prediction of freckle formation: mathematically derived criterions and predictions based on numerical solution of the continuity equations. ${ }^{25}$ The first criterions developed were based on functional forms involving controlling operational parameters that could be measured or established in industrial practice like the gradient, melting rate or the local solidification time. ${ }^{3}$ M. C. Flemings and G.E. Nereo ${ }^{26}$ derived a mathematical description of macrosegregation and postulated the local solute redistribution equation, which constituted the foundation for the Flemings criterion presented as the inequality 1. Basically, a freckle channel will grow if the liquid flow from colder to hotter regions is faster that the rate at which crystals grow. ${ }^{27}$

$$
\frac{\vec{v} \cdot \nabla T}{\varepsilon} \leq-1
$$

Flemings et al. proposed criterion presents a physical explanation of the remelting effect of the established convective flow, but requires relating the interdendritic flow with its causes. That is why their expression have been considered as determining if a channel will evolve rather than predicting its nucleation. ${ }^{10,15}$ Currently, the most accepted freckling criterion is the one based on the maximum Rayleigh number concept. The Rayleigh number may be defined as the ratio between the buoyancy and the viscous dissipation forces at a point of interest. In the particular 
case of convection in the mushy zone, the driving force is due to compositional buoyancy and the viscous dissipation is related to flow impedance through the dendritic array. The Rayleigh number based criterion has the advantage that incorporates the thermal and compositional effects over the freckling tendency. The threshold value of the Rayleigh number defining between the freckling and non freckling conditions is denominated critical Rayleigh number, and it is theoretically believed to be universally applicable. If the maximum Rayleigh number for a determined set of conditions is below the critical value, freckles are not expected to form, but it is possible that the system could have a maximum Rayleigh number higher than the critical value and yet no freckles being formed. ${ }^{28}$ The Rayleigh number follows from the dimensionless mathematical description of convection ${ }^{29}$ but there is no consensus in its applicable formula for the freckling prediction application. By reviewing a series of Rayleigh number forms proposed, Yang ${ }^{30}$ remarked that the major difference between them was the meaning given to the characteristic length of the system considered. Beckermann et al. ${ }^{31}$ developed a criterion based on the maximum Rayleigh number using a form similar to the one deduced by Worster ${ }^{12}$ and expressed by equation 2 , where $\left(\Delta \rho / \rho_{0}\right)$ is the relative density inversion, $\mathrm{g}$ is the gravity, $\mathrm{K}$ is the averaged permeability, $\mathrm{h}$ is the distance measured from the top of the mush to the point of interest, $\alpha$ is the thermal diffusivity and $v$ is the kinematic viscosity.

$$
R a=\frac{\left(\Delta \rho / \rho_{0}\right) \cdot g \cdot K \cdot h}{a \cdot v}
$$

To validate their formulation, full numerical simulations were performed with the parameters used on each of the upward solidification experiments by Pollock and Murphy ${ }^{32}$ on single crystal superalloy SX-1, and the local Rayleigh numbers calculated at the initiation sites of fully simulated freckles were considered as the critical values. A critical value of approximately 0.25 was determined from the whole set of simulations with vertical domains. The difference between the threshold obtained from the experiments and the simulations was considered within the uncertainties related with the experimental parameters. Additionally, it was shown that the inclination angle with respect to gravity decreased the threshold values. ${ }^{33}$ Beckermann and coworkers contribution has the disadvantage that it assumes an isotropic permeability and its validation assumed a priori that the freckles should form in the top of the mush. Furthermore, the isotropic mean permeability was calculated using an average over the solid fraction along a vertical path, which tailors their expression to the case of freckling due to density inversion, and 
makes it not as suitable for the freckles in VAR ingots. Yang et al. ${ }^{30}$ presented a freckle criterion for upward directional solidification of alloys. Their proposed form is given by equation 3 , where $\Delta \rho$ represents the relative density change as defined above, $g$ is the gravity, $\Pi$ is the total averaged permeability, $v$ is the liquid kinematic viscosity, $f_{L}$ is the liquid volume fraction and $R$ is the crystal growth rate.

$$
R a=\frac{(\Delta \rho) \cdot g \cdot \Pi}{v \cdot f_{L}} \cdot \frac{1}{R}
$$

Permeability was assumed as a scalar with a magnitude equal to the value calculated using Poirier's formula for permeability component in the direction parallel to the primary dendrites. ${ }^{34}$ In order to take into account the need of collective motion of liquid along the vertical path, an integration method similar to the one used by Beckermann was applied. The proposed criterion was evaluated using directional solidification experimental data for $\mathrm{Pb}-\mathrm{Sn}, \mathrm{Pb}-\mathrm{Sb}$ and $\mathrm{Pb}-\mathrm{Sn}-\mathrm{Sb}$ alloy systems and it provided an improvement on the resolution for the freckling prediction with respect to the Rayleigh number forms reviewed, but a critical Rayleigh number value could not be defined because of overlapping of various experimental observations between the freckle-no freckle conditions. In a latter work, Yang et al. extended their form to include the effect of the tilt of the solidification front and performed horizontal solidification experiments for verification. ${ }^{23}$ The modified form had the weakness that it included the angle that the velocity vector formed with the isotherms, which is not known before hand, and makes it difficult to establish an accurate prediction of freckling. Auburtin et al. ${ }^{25}$ evaluated the effect of solidification front tilt with respect to the gravity vector. To include this geometrical effect, they modified the original form proposed by Sarazin and Hellawell ${ }^{35}$ by replacing the characteristic length with an expression including the permeability of the mushy zone. The modified criterion obtained is presented in equation 4 , where $D t$ is the thermal diffusivity, $\lambda_{1}$ is the primary dendrite arm spacing and $K$ is the permeability in the vertical direction ${ }^{36}$, which is related to the tilt angle $\alpha$ of the solidification front with respect to the horizontal plane, and the parallel $\left(K_{Y}\right)$ and perpendicular $\left(K_{X}\right)$ components of the permeability by equation 5 . Validation experiments were performed using a directional solidification furnace capable of inducing a tilted solidification front forming an angle between 0 and 35 degrees with the horizontal. 


$$
\begin{gathered}
R a=\frac{g \cdot \frac{\partial \rho}{\partial z}}{v \cdot D_{T}} \cdot\left[\lambda_{1}\left(\frac{K}{K_{Y}}\right)\right]^{4} \\
K=\frac{1}{\frac{\sin ^{2} \alpha}{K_{X}}+\frac{\cos ^{2} \alpha}{K_{Y}}}
\end{gathered}
$$

Auburtin et al. proposed criterion provided good resolution in the prediction of freckling for the experimental results. However, their criterion shows a monotonically increasing trend with increasing the tilt angle, even when the flow driving force should decrease at high tilt angles. The critical Rayleigh number values for the different alloys were found to be in a range between 0.65 and 0.95 . The deviation from unity was attributed to secondary features of the dendrite morphology. ${ }^{37}$ The main objectives of the work presented here were to develop a Rayleigh number based criterion for freckling prediction on superalloy remelt ingots, which by including the effect of tilt angle, provides a better resolution between the predicted freckled - non freckled conditions, and to evaluate its performance against experimental data available in the literature.

\section{Development of the Proposed Freckle Criterion}

It is considered that the mush three-dimensional dendritic array of a solidifying VAR ingot is properly described by a two-dimensional region lying over a diametral plane. Due to the concave geometry of the mush, the solidification front forms a tilt angle $\theta$ with respect to the horizontal plane that is function of the position along the radius as represented in Fig. 1. It is assumed an elementary volume in the mushy layer with the same characteristics as the one defined by M.C. Flemings and G.E. $\mathrm{Nereo}^{26}$ i.e. sufficiently large to permit averaging of the microscopic properties and having a liquid fraction within, that is exactly equal to the local average, but still it is sufficiently small to be considered as a differential element. The permeability in point $O$ is mathematically described by a second order tensor, which when referred to a coordinate system $\left(O X_{1} X_{2}\right)$ that coincides with its principal directions, parallel and perpendicular to the primary dendrites, its non-zero components are the principal values (equation 6) that can be defined by experimentally developed functional forms. Those equations

formulated by Poirier ${ }^{34}$ are reported as equations 7 and 8, and they involve the volume fraction liquid $f_{L}$, the arm spacing of primary $\left(\lambda_{1}\right)$ and the arm spacing of secondary dendrites $\left(\lambda_{2}\right)$. 


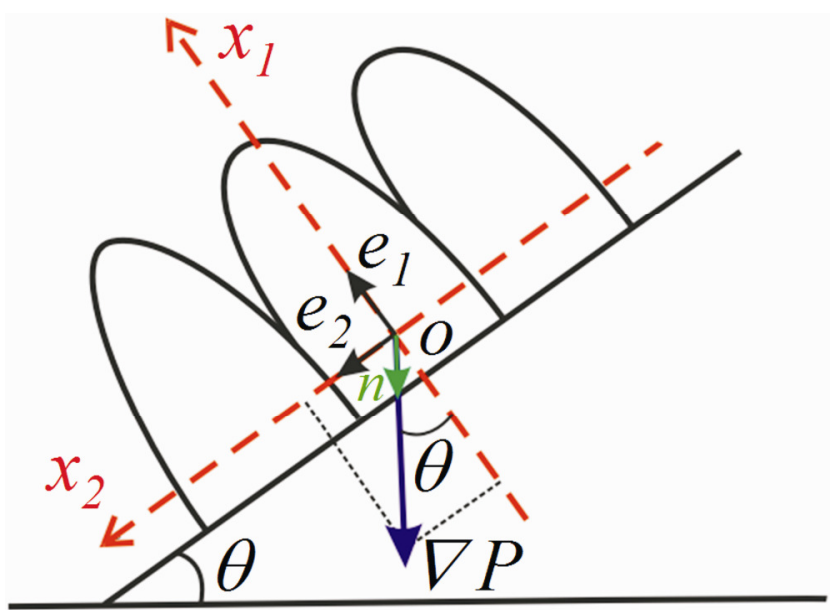

Fig. 1 - Schematic representation of the mushy layer with tilted solidification front

$$
\begin{gathered}
K=\left[\begin{array}{cc}
K_{I} & 0 \\
0 & K_{I I}
\end{array}\right] \\
K_{I}=3.75 \cdot 10^{4} \cdot f_{L}^{2} \cdot \lambda_{1}^{2} \\
K_{I I}=3.62 \cdot 10^{3} \cdot f_{L}^{3.34} \cdot \lambda_{1}^{0.699} \cdot \lambda_{2}^{2.73}
\end{gathered}
$$

Expressions relating the interdendritic spacing of primary and secondary dendrites with the cooling rate ${ }^{38}$ were used as presented in equations 9 and 10 . The interdendritic spacing units are $[\mathrm{m}]$, and the cooling rate $\left(\mathrm{G}^{*} \mathrm{R}\right)$ should be given in $[\mathrm{K} / \mathrm{s}]$.

$$
\begin{aligned}
& \lambda_{1}=\frac{150 \cdot 10^{-6}}{(G \cdot R)^{0.33}} \\
& \lambda_{2}=\frac{40 \cdot 10^{-6}}{(G \cdot R)^{0.42}}
\end{aligned}
$$

No solidification shrinkage was considered and the only driving force is the solutal buoyancy, proportional to the difference between the density at the liquidus temperature $\left(\rho_{0}\right)$ and the local density at point $O(\rho)$, represented by $\Delta \rho$. Let a potential driving force vector be defined as the gradient density multiplied by the length $\Delta z$ measured from the tip of the dendrites to the point $O$ with magnitude equal to $\Delta \rho$ and acting along the $X_{1}$ axis. The inner product of the potential driving force vector (perpendicular to the isodensity lines) and the gravity acceleration vector $g$ will give the magnitude for the pressure gradient vector as given in equation 11 . The pressure 
gradient vector acts in the direction of unit vector $\boldsymbol{n}$ pointing vertically downwards as shown in Fig. 1.

$$
|\vec{\nabla} P|=\Delta \rho \overrightarrow{e_{1}} \cdot g \vec{n}=\Delta \rho g \cos \theta
$$

Using the Darcy law, ${ }^{39}$ the interdendritic velocity was obtained by equation 12.

$$
\vec{v}=\left(\frac{1}{\mu f_{\mathrm{L}}}\right) \bar{K} \cdot \vec{\nabla} P=\left(\frac{\Delta \rho g \cos \theta}{\mu f_{\mathrm{L}}}\right)\left[\begin{array}{c}
K_{I} \cos \theta \\
K_{I I} \sin \theta
\end{array}\right]
$$

It is assumed that the Darcy law of flow through porous media applies in a representative elementary volume in the mushy layer following the methodology proposed by S. Ganesan and D.R. Poirier. ${ }^{40}$ By using the Flemings criterion, the proposed local Rayleigh number form is obtained by calculating the ratio between the interdendritic velocity vector magnitude and the growth rate as follows:

$$
R a_{-} p=\frac{\vec{v} \mid}{R}=\left(\frac{\Delta \rho g \cos \theta}{\mu f_{L}}\right) \frac{\left(K_{I}^{2} \cos ^{2} \theta+K_{I I}^{2} \sin ^{2} \theta\right)^{\frac{1}{2}}}{R}
$$

Where $\mu$ is dynamic or absolute viscosity of the interdendritic liquid, $R$ is the growth rate, and $K_{I}$ and $K_{I I}$ are the permeability components in the parallel and perpendicular directions to the primary dendrites respectively, as were defined above. The Rayleigh number form is obtained by a similar method used by Yang ${ }^{30}$, with major differences being that in this work the gradient of the pressure is derived differently, the effect of the tilted solidification front is included without the need to know a priori the direction of the velocity vector and most importantly, the two dimensional anisotropic characteristic of the permeability tensor is preserved throughout the formulation. The simpler, but adequate ${ }^{11}$ case of equilibrium between the solid dendrites and the interdendritic liquid was assumed in order to determine the solidification path, the composition and the density of the interdendritic liquid as functions of temperature using JMatPro® software. The calculations for the components $\mathrm{K}_{\mathrm{I}}$ and $\mathrm{K}_{\mathrm{II}}$ were performed directly using the local fraction liquid determined with JMatPro®. No assumption was used about a particular cooperative motion path in order to maintain both the isotropy for the permeability and the generalized application for the resulting Ra number form. To preserve simplicity and emphasize the differences of performance attributed to the geometric component, the viscosity of the 
interdendritic liquid was considered constant with the same value for all alloys and temperatures. The generalized value adopted here was $\mu=0.004$ in $\left[\mathrm{Kgm}^{-1} \mathrm{~s}^{-1}\right]$. The effect of variable viscosity is critical and its accurate determination should be considered for future work.

\section{Evaluation of the Proposed Form}

The criterion based on the maximum proposed local Rayleigh number was evaluated using experimental data published by two different research groups involving directional solidification of both commercial and model Ni-based superalloys. The experiments included variations on the tilt angle, temperature gradient at the mush - bulk liquid boundary and on the growth rate of the solid crystals. The local Rayleigh numbers were calculated using equation 13 for all experiments. The compositions of the alloys used for the evaluation are presented in table 1, while the values for the experimental independent variables and the resulting freckle or no-freckle conditions are presented in table 2. It is suggested to refer to the original publications for complete details about the experimental procedures. ${ }^{23,37,38}$

Table I. Alloy composition for the directional solidification experiments reviewed.

\begin{tabular}{|l|l|l|l|l|l|l|l|}
\hline & RN902 & RN903 & RN5010 & R4007 & \multicolumn{1}{|c|}{718} & $718 \mathrm{LSi}$ & $718 \mathrm{HSi}$ \\
\hline $\mathrm{Ni}$ & 54.43 & 70.57 & 73.93 & Bal. & 53.94 & 54.03 & 53.41 \\
\hline $\mathrm{Cr}$ & 19.95 & 20.09 & 19.98 & 18.0 & 18.48 & 18.0 & 18.58 \\
\hline $\mathrm{Al}$ & - & - & - & 0.5 & 0.37 & 0.5 & 0.38 \\
\hline $\mathrm{Ti}$ & - & - & - & 1.0 & 1.08 & 1.0 & 0.90 \\
\hline $\mathrm{Mo}$ & - & - & - & 3.0 & 3.07 & 3.0 & 2.86 \\
\hline $\mathrm{Nb}$ & 7.13 & 8.57 & 6.36 & 5.0 & 4.66 & 5.0 & 4.05 \\
\hline $\mathrm{C}$ & 0.014 & 0.004 & 0.005 & - & - & 0.008 & 0.62 \\
\hline $\mathrm{Si}$ & 0.03 & 0.01 & 0.01 & - & 0.23 & 0.007 & - \\
\hline $\mathrm{Fe}$ & 17.75 & 0.69 & 0.06 & 18.0 & 18.63 & 18.45 & 19.45 \\
\hline
\end{tabular}

Table II. Data reported for directional experiments

\begin{tabular}{|c|c|c|c|c|}
\hline & $\mathbf{G}(\mathrm{C} / \mathrm{mm})$ & $\mathbf{R}(\mathrm{mm} / \mathrm{min})$ & Tilt angle $\boldsymbol{\alpha}$ & Freckle \\
\hline \multirow{4}{*}{ RN902 } & 6.12 & 1.72 & 50 & $\mathrm{~N}$ \\
\cline { 2 - 5 } & 4.09 & 1.5 & 50 & $\mathrm{Y}$ \\
\cline { 2 - 5 } & 3.19 & 1.2 & 50 & $\mathrm{Y}$ \\
\cline { 2 - 5 } & 2.92 & 0.8 & 50 & $\mathrm{Y}$ \\
\hline \multirow{4}{*}{ RN903 } & 4.8 & 1.36 & 52 & $\mathrm{~N}$ \\
\cline { 2 - 5 } & 4.39 & 1.3 & 52 & $\mathrm{~N}$ \\
\cline { 2 - 5 } & 2.97 & 1.17 & 52 & $\mathrm{~N}$ \\
\cline { 2 - 5 } & 2.51 & 1.0 & 52 & \\
\hline
\end{tabular}




\begin{tabular}{|c|c|c|c|c|}
\hline & 2.28 & 0.82 & 52 & $\mathrm{Y}$ \\
\hline & 2.53 & 0.6 & 52 & $\mathrm{Y}$ \\
\hline \multirow[b]{2}{*}{ RN5010 } & 2.14 & 2.25 & 82 & $\mathrm{~N}$ \\
\hline & 3.5 & 0.34 & 34 & $\mathrm{Y}$ \\
\hline \multirow{4}{*}{ R4007 } & 2.19 & 2.96 & 33 & $\mathrm{~N}$ \\
\hline & 1.40 & 2.70 & 33 & $\mathrm{Y}$ \\
\hline & 1.02 & 2.25 & 33 & $\mathrm{Y}$ \\
\hline & 0.95 & 1.59 & 33 & $\mathrm{Y}$ \\
\hline \multirow{5}{*}{718} & 1.0 & 6.0 & 35 & $\mathrm{~N}$ \\
\hline & 2.6 & 1.0 & 35 & $\mathrm{Y}$ \\
\hline & 1.0 & 6.0 & 20 & $\mathrm{~N}$ \\
\hline & 2.6 & 1.0 & 20 & $\mathrm{Y}$ \\
\hline & 1.4 & 1.0 & 0 & $\mathrm{Y}$ \\
\hline \multirow{5}{*}{718 LSi } & 1.5 & 6.0 & 35 & $\mathrm{~N}$ \\
\hline & 2.9 & 1.0 & 35 & $\mathrm{Y}$ \\
\hline & 1.5 & 6.0 & 20 & $\mathrm{~N}$ \\
\hline & 2.9 & 1.0 & 20 & $\mathrm{~N}$ \\
\hline & 2.0 & 1.0 & 0 & $\mathrm{~N}$ \\
\hline \multirow{5}{*}{718 HiSi } & 0.9 & 6.0 & 35 & $\mathrm{~N}$ \\
\hline & 3.5 & 1.0 & 35 & $\mathrm{Y}$ \\
\hline & 0.9 & 6.0 & 20 & $\mathrm{~N}$ \\
\hline & 3.5 & 1.0 & 20 & $\mathrm{Y}$ \\
\hline & 3.1 & 1.0 & 0 & $\mathrm{Y}$ \\
\hline
\end{tabular}

\section{Discussion}

Resolution performance

After calculation of the local Rayleigh number for all experiments, the maximum values were gathered and plotted against the corresponding alloy as shown in Fig. 2. It is observed that for all the alloys was possible to clearly resolve the conditions of freckle -No freckle. The included inset details two experimental values which are very close for alloy $718 \mathrm{LSi}$, but still there is no overlapping of the freckle-no freckle conditions. Additionally, the Rayleigh form using the methodology proposed shows that a horizontal line could be drawn at approximately 0.14 value, below which no freckle are expected to occur for all the alloys evaluated, and it could be considered as the critical Rayleigh value. Its magnitude is fairly close to 0.25 proposed by Beckerman et al. for superalloys ${ }^{31}$, taking into consideration all the involved uncertainties in the experimental measurements, the assumptions on viscosity, thermodynamical equilibrium in the 
solid-liquid interface and the experimental nature of the equations used to calculate the dendritic arm spacing and the permeability components.

$\underline{\text { Tilted solidification front effect on the local Rayleigh number and nucleation sites }}$

Example results can be observed in Fig. 3 for experiments performed using model alloy RN902. The local Rayleigh number is increased, by the lower cooling rate for all positions in the mush. Different thermal parameters with the same tilt angle do not produce a noticeable effect over the fraction liquid where the maximum Rayleigh number occurs, which should correspond to the site with the highest potential for channeling nucleation in the mush. Additionally, it is concluded for the example cases of Fig. 3 that the local Rayleigh number range containing the threshold critical value is defined by the limiting maximum values of approximately 0.08 and 0.18 . Fig. 4 shows the Rayleigh number variation with $f_{L}$ for two experiments performed on superalloy IN718LSi.

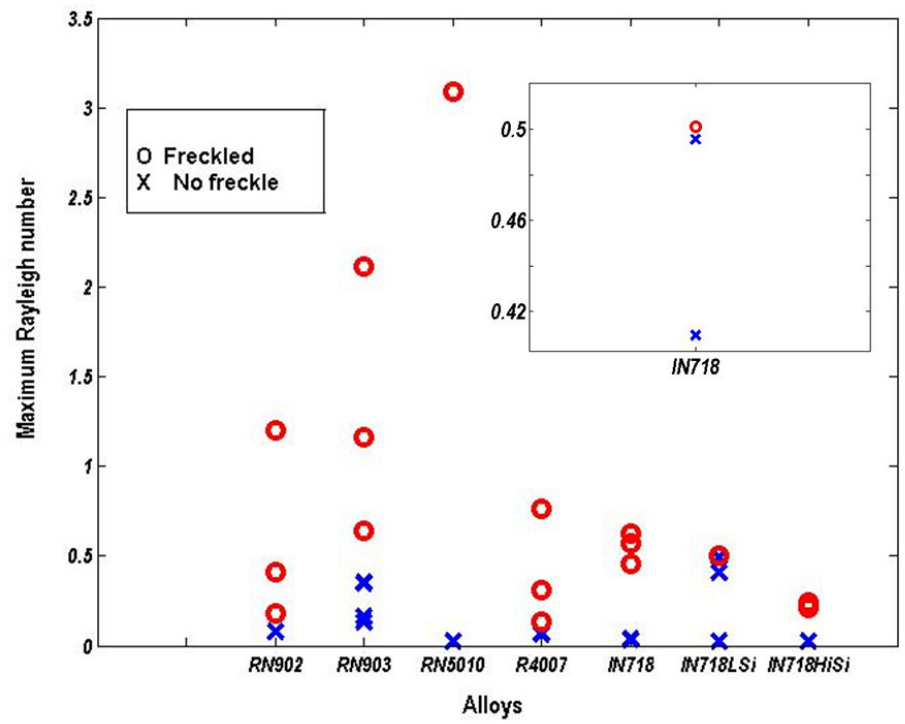

Fig. 2 Maximum Rayleigh number vs. Alloys (No permeability averaging)

Both of the local Rayleigh number curves show a rather abrupt variation of curvature at a liquid fraction of 0.13 related to a change in the solidification path due to the precipitation of laves phase. The thermal conditions for the two experiments were the same, so the morphology of dendrite arms between the two cases should be the same, while the tilt angles were 35 and 20 degrees. It is evident that the tilt angle change results in a geometrical condition with increased permeability, due to a higher contribution of the transverse direction component that elevates the local Rayleigh number in the mush and favors the freckle formation. The potential nucleation 
site for channeling is shifted to a higher position in the mush where the solid fraction is lower, and a weaker driving force is sufficient to drive an unstable convective flow. The resolving capability between the freckle - No freckle of the form proposed when applied to experiments like the ones presented in Fig. 4 could not be available if the permeability is considered as a scalar or as an isotropic second order tensor.

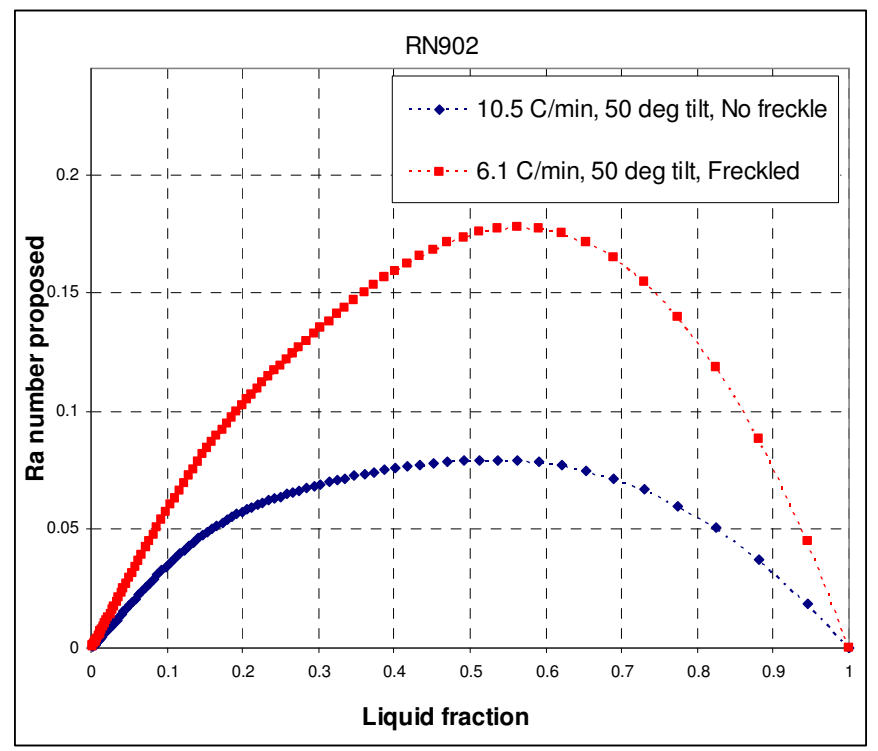

Fig. 3 - Local Rayleigh number variation with $f_{L}$ for model alloy RN902

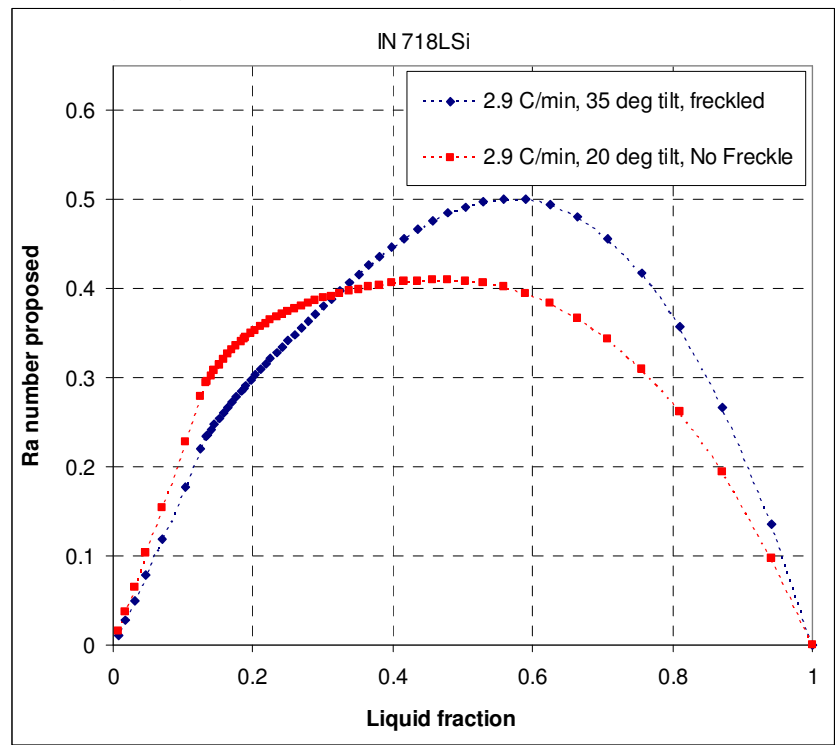

Fig. 4 - Local Rayleigh number proposed vs. fL for alloy IN718LSi

\section{Conclusions and Future Work}

A freckling criterion based on the maximum local Rayleigh number was proposed for the remelting of Ni-based superalloys. The calculated maximum Rayleigh number values using 
experimental data available in the literature provided a clear separation between the observed freckle - No freckled conditions, and the critical Rayleigh number deduced was approximately 0.14 , which is in accordance with a previously proposed value for superalloys derived by a completely different approach. ${ }^{31}$ This result evidences the need of further understanding of the phenomenon, since a critical value should be universal and theoretically near unity. For example, it's fundamental to keep in mind that the viscosity, which in this work was assumed constant, plays a strong role on the stability. If the variation of the liquid viscosity is included by JMatPro calculation, for the alloy RN5010, the Rayleigh number proposed is decreased for all fraction liquid values and in fact, because the viscosity calculated values are greater than the one assumed, the maximum local Rayleigh number is slightly shifted to the lower fraction liquid region where the driving force is greater. Fig. 5 shows the described effect.

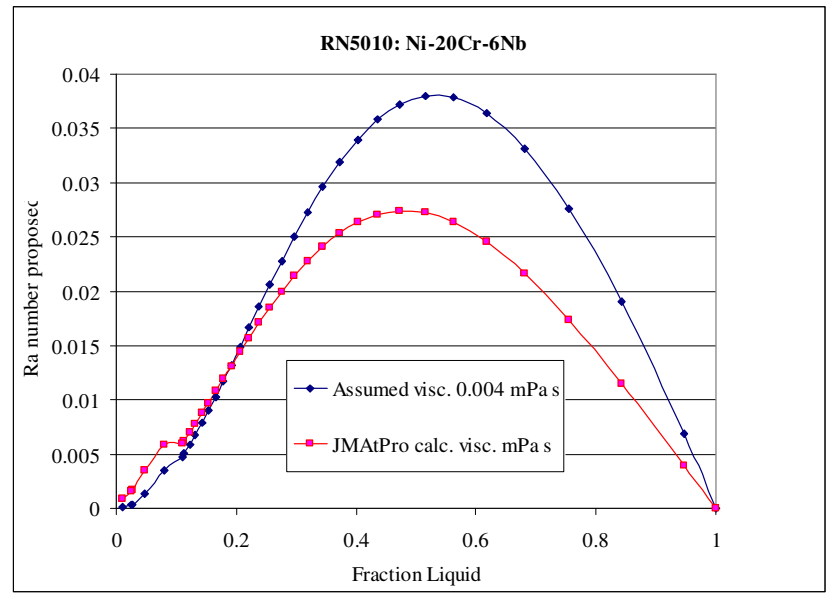

Fig. 4 - Effect of viscosity assumption over the Local Rayleigh number proposed vs. fL for alloy RN5010

The effect of a tilted solidification front was addressed and it was demonstrated that under the same thermal conditions of solidification, a tilted solidification front induces a higher freckling potential. Further research is needed to clarify the details of the freckle formation mechanism in VAR ingots, and to minimize the influence of uncertainties and critical assumptions incorporated over the accuracy of the Rayleigh number criterion predictions.

\section{References}

1 R. Schwant, S. Thamboo, L. Yang, M. Norra: Superalloys 718, 625, 706 and Derivatives 2005, ed. E. A. Loria, TMS, Warrendale, PA, 2005,pp.15-24 
2 X. Wang, R. M. Ward, M.H. Jacobs and M. D. Barrat: Metal. Mater. Trans. A, 2008, vol. 39A, pp.2981-2989

3 S.M. Copley, A. F. Giamei, S. M. Johnson and M. F. Hornbecker: Metal. Trans., 1970, vol. 1, pp. $2193-2204$

4 D.G. Nielson and F. P. Incropera: Int. J. Heat Mass Transfer, 1991, Vol. 34, pp. 1717-32

5 S. Tait and C. Jaupart: J. Geophys. Res. , 1992, Vol. 97, pp. 6735-56

6 P. D. Genereux and C. A. Borg: Superalloys 2000, eds., K. A. Green, T.M. Pollock and R. D. Kissinger, TMS, Champion, PA, 2000, pp.19-27

7 A.K. Sample and A. Hellawell: Metall. Trans. A, 1984, Vol. 15, pp. 2163-73

8 D.G. Nielson and F.P Incropera: Int. J. Heat Mass Transfer, 1993, Vol. 36, pp. 489- 505

9 D.G. Nielson and F.P. Incropera: Exp. Heat Transfer, 1993,Vol. 6, pp. 131-55

10 C. Frueh, D.R. Poirier, R.G. Erdmann, S. D. Felicelli: Mater. Sci. Eng. A, 2003, Vol. 345, pp.72-80

11 M.C. Schneider and C. Beckermann: Int. J. Heat Mass Transfer, 1995, Vol. 38, pp.3455-73

12 M.G. Worster: J. Fluid Mech., 1992, Vol. 237, pp. 649-69

13 P.W. Emms: J. Eng. Math., 1998, Vol.33, pp.175-200

14 S. Tait, K. Jahrling, C. Jaupart: Nature, 1992, Vol. 359, pp.406-08

15 A. Mitchell: Mater. Sci. Eng. A, 2005, 413-414, pp. 10-18

16 I. G. Hwang and C. K. Choi: Korean J. Chem. Eng., 2008, Vol. 25, pp. 199-02

17 James H. Van Den Avyle, John A. Brooks, and Adam C. Powell: JOM,1998,vol.50(3), pp. 22-25

18 P. Auburtin, S. L. Cockcroft, A. Mitchell, A.J. Schmalz: Superalloys 718,625, 706 and various Derivatives, E. A. Loria ed., TMS, Pittsburgh, PA, 1997, pp.47-54

19 P. Auburtin, S. L. Cockcroft, A. Mitchell: Superalloys 1996, R.D. Kissinger, D.J. Deye, D.L. Anton, and A.D. Cetel, eds., TMS, Champion, PA, 1996, pp. 443-50

20 A. D. Patel, R. S. Minisandram and D. G. Evans: Superalloys 2004, K.A. Green, T.M. Pollock, H. Harada, T.E. Howson, R.C. Reed, J.J. Schirra, and S. Walston, eds., TMS, Warrendale, PA, pp. 917-924

21 D. Zagrebelnyy and M. J. M. krane: Metall. Mater. Trans. B, 2009, Vol. 40, pp. 281- 288

22 L. Yuan, P. D. Lee, G. Djambazov and K. Pericleous: International symposium on liquid metal processing and casting, 2009, eds. Peter Lee, Alec Mitchell, and Rod Williams, TMS, 2009, Santa Fe, New Mexico, pp. 39-46

23 W.H. Yang, J. J. deBarbadillo, K. Morita, T. Suzuki, W. Chen, K. M. Chang: JOM, 2004, Vol. 56 (9), pp. 56-61

24 K. Kajikawa, T. Sato and H. Yamada: International symposium on liquid metal processing and casting, 2009, eds. Peter Lee, Alec Mitchell, and Rod Williams, TMS, 2009, Santa Fe, New Mexico, pp. 327-335

25 P. Auburtin, T. Wang, S.L. Cockcroft and A. Mitchell: Metall. Mater. Trans. B, 2000, vol.31B, pp.801-811

26 M.C. Flemings and G.E. Nereo: Trans. TMS-AIME, 1967, vol.239, pp.1449-61. 
27 R. Mehrabian, M. Keane and M.C. Flemings: Metall. Trans., 1970, vol.1, pp. 1209-20.

28 M. G. Worster: Annu. Rev. Fluid Mech. , 1997, Vol. 29, pp. 91-122

29 M. G. Worster: J. Fluid Mech., vol. 224. 1991, pp. 335-359

30 W. H. Yang, W. Chen, K. M. Chang, S. Mannan, J. deBarbadillo: Metall. Mater. Trans. Vol. 31A, 2001, pp. 397406

31 C. Beckermann, J. P. Gu and W. J. Boettinger: Metall. Mater. Trans. A, 2000, Vol. 31, pp. $2545-57$

32 T.M. Pollock and W.H. Murphy: Metall. Mater. Trans. A, 1996, Vol. 27, pp. 1081-1094

33 J. C. Ramirez and C. Beckermann: Metall. Mater. Trans. A, 2003, Vol. 34, pp. 1525-36

34 D.R. Poirier: Metall. Trans B, 1987, vol.18B, pp.245-55

35 J. R. Sarazin and A. Hellawell: Metall. Trans. A, 1988, vol. 19A, pp.1861-1871

36 A. E. Scheidegger: The physics of flow through porous media, revised edition, The MacMillan Company , New York, 1960, pp.76-79

37 P. Auburtin, S.L. Cockcroft, A. Mitchell and T. Wang: Superalloys 2000, eds. T. M. Pollock, R. D. Kissinger, R. R. Bowman, K. A. Green, M. McLean, S. Olson and J. J. Schirra, TMS, 2000, Champion , PA, pp.255-261

38 P. Auburtin: PhD. Thesis, University of British Columbia, Vancouver, BC, Canada, Aug. 1998.

39 M.S. Bhat, D.R. Poirier, J.C. Heinrich and D. Nagelhout: Scripta Metall. Mater., 1994, vol. 31, pp.339-344

40 S. Ganesan and D.R. Poirier: Metall. Trans. B, 1990, vol. 21B, pp. 173-181 